\title{
Protokoll der Diskussion in der Redaktionskonferenz zum Aufsatz von Scharrer/Schütte
}

1. Da die Veröfentlichung des Aufsatzes von Scharer/Schütte innerhalb der PROKLA-Redaktionskonferenz aukerordentlich kontrovers diskutiert wurde, sollen hier die Argumente zusammengefaßt werden, die gegen den Aufsatz geäußert wurden. Die kritischen Einwände spiegeln also keineswegs die einhellige Meinung der Redaktionskonferenz. wider. Sie sind auch nicht ausschlieklich gegen den Artikel von Scharrer/Schütte gerichtet, sondern wenden sich vor allem gegen mögliche Fehlentwicklungen einer politischen Richtung, die durchaus wesentliche Probleme der Geschichte der Arbeiterbewegung aufgreift, gerade aber in ihren moralischen und teilweise subjektivistischen Verküzungen historische Lernprozesse der Arbeiterklasse, die auf die reale Entwicklung des Kapitalverhätnisses bezogen sein müssen, sollen sie zur Entwicklung des Klassenkamptes beitragen, erschwert.

Die Frage nach den politischen Konsequenzen einer Veröffentlichung der in der Kritik am Projekt Klassenanalyse entwickelten Positionen verweist auf ein Problem der PROKLA, das nicht zufällig am Aufsatz von Scharrer/Schüte deutlich wird: den Widerspruch der objektiven politischen Aukenwirkung der PROKLA einerseits und der noch unausdiskutierten politischen Position. Stokrichtung und Funktion der PROKLA inmerhalb der Redaktionskonferenz andererseits. Wenn der Redaktionskonferenz dieses Problem nicht bewukter als bisher wird, besteht die Gefahr, bewulktseinsbildend zu wirken, ohne jedoch die Resultate dieses Prozesses aufnehmen und politisch weitertreiben zu können.

Die Redaktionskonterenz hat sich nach längerer Diskussion für die Veröffentlichung entschieden, da Scharrer/Schüte wesentliche Fragen des Zusammenhangs von Klassenkampt, Klassenbewuhtsein, Organisation und Partei aufwerfen, die von den existierenden Studentenparteien und der DKP länst tabuisiert werden, um sich stattdessen dogmatisch auf ein vermeintlich historisch legitimiertes oder gar als aus der allgemeinen Analyse der kapitalistischen Gesellsehaft mmittelbar ableitbares Parteimodell festzulegen.

Die Kritik der Redaktionskonferenz am Anfang zielt vor allem auf einen Punkt: den aus der Geschichte der Studentenbewegung bezogenen moralischen Anspruch auf Emanzipation an die Organisation des Proletariats statt einer historisch-materialistischen Ableitung des Zusammenhangs von Kapitalentwicklung, Klassenkampf, Klassenbewuntsein, Organisation und Partei.

2. Mit ihrer abstrakten ahistorischen Leninismuskritik, der Unterschlagung der Leninschen politischen Lemprozesse und der historischen Bedeutung Lenins in der Verknüptung eines materialistisch nicht erklärten Despotismusvorwurfs liefern Scharrer/Schüte sicherlich ungewollt denjenigen nüzliche Argumente, die sich schon immer mit dem abstrakten Despotismusvorwurf gegenüber dem "Sowjetkomnunismus" zutrieden gaben, um damit ihren Antikommunismus ideologisch zu 
rechtfertigen. Die Rekonstruktion einer sozialdemokratischen Ideologie des demokratischen Sozialismus, wie sie in der aktuellen Absetzung von den ,Linksradikalen" durch die DKP und Teile der Jungsozialisten erfolgt, erfährt durch eine solche abstrakte Kritik wesentliche Unterstützung.

3. Dem berechtigten Angriff auf den Logizismus des Projekts Klassenanalyse liegt eine aufgrund einer einseitigen Marx rezeption und moralischer Postulate entstandene Gegenidentifikation zugrunde, aus der die Stellung zu Partei, Gewerkschaften, Klassenbewußtsein und Klassenkampf deduziert wird. Der ihrer Kritik zugrundeliegende richtige Anspruch, die Entstehung von Klassenbewußtsein, Klassenkampf und Organisation aus der realen historischen Bewegung herzuleiten, wendet sich gegen die Autoren selbst, da er an keiner Stelle inhaltlich gefült wird. Vielmehr unterlaufen sie ihren zutreffenden Hinweis, aus der Marxschen Darstellung der allgemeinen Bewegungsge se tze der kapitalistischen Gesellschaft lasse sich keine spezifische Form der politischen Organisation des Proletariats ableiten, indem bei der Marxschen Behandlung der englischen Gewerkschaften durch Scharrer/Schiitte der Eindruck entstehen kann, als habe Marx die gewerkschaftliche Organisationsform als die aus der realen Entwicklung entstehende, einzige adäquate politische Organisation der Arbeiterklasse abgeleitet.

4. Der in den Feuerbachthesen entwickelte emphatische Begriff der Praxis als Vermittlungsprozefo von Subjekt und Objekt wird den Verfassern zum voluntaristischen Postulat, das die Marxsche Weiterentwicklung des Praxisbegriffs zur Konstituierung von Klassen bewußtsein innerhalb des organisierten Klassenkampfs vernachlässigt.

Da in dem Aufsatz keine Verknupfung von Klassenkampf, Klassenbewußtsein, Massenorganisation, Partei und sozialer Emanzipation geleistet wird, gerät die richtige Betonung sozialer Emanzipation gegenüber einem letztlich abstrakten, objektivistischen Politik- und Kampfbegriff des orthodoxen Marxismus vor allem der 2. Internationale tendenziell zum subjektivistischen Anspruch auf individuelle Befreiung.

Die psychische Verkrüppelung der Individuen in der bürgerlichen Gesellschaft sowie die Möglichkeiten sozialer Emanzipation werden nicht aus der Struktur und der spezifischen Form der Vergesellschaftung der Arbeit im Prozeß der Reproduktion und Akkumulation des Kapitals hergeleitet, sondern verschiedene Momente der Verelendung werden unverbunden, so wie sie das Kapital an der Oberfläche der bürgerlichen Gesellschaft produziert, aufgenommen und im Begriff der Emanzipation versucht aufzuheben. ohne daß dabei der revolutionäre Umschlag aus der Entwicklung des Kapitalverhältnisses, des Klassenkampfs und den daraus erwachsenden Bewußstseinsformen klar würde. Die Entwicklung des Widerspruchs von bürgerlicher Sozialisation und der Konstituierung von Klassenbewuß tsein kann nicht durch individualpsychologische Ansätze begriffen werden, sondern nur, wenn es gelingt, Sozialisation, psychische und Bewußtseinsentwicklung im Zusammenhang der kapitalistischen Form der Vergesellschaftung zu vermitteln, sowie mögliche Ansätze ihrer Aufhebung aus den auf die Umwälzung der bestehenden Verhältnisse gerichteten Klassenkämpfen und̀ Organisationsformen anzugeben. 
Die Aufhebung der Isolierung der Lohnabhängigen und die Entstehung von Klassenerkenntnis ist in den Begriffen von Praxis und Emanzipation zu kurz gegriffen. Klassenerkenntnis und die Befreiung des Individuums können sich nur im organisierten Klassenkampf und der bewußten Vergesellschaftung von Arbeit politisch wirksam entfalten. Die Betonung des emanzipativen Charakters des politischen Kampfes, die Auflösung instrumenteller Vorstellung von Klassenkampf als simpler Zweck-Mittel-Relation, der Hinweis auf den notwendigen Abbau von Hierarchisierungen, Befehls- und Gehorsamsmechanismen der Entscheidungsstrukturen innerhalb der Klassenorganisation verweisen auf einen richtigen Zusammenhang: daß de Aufbau des Sozialismus als sozialer Revolution im Vermittlungszusammenhang der bewußten Vergesellschaftung der Arbeit nicht von den Formen und Mitteln des Klassenkampfs innerhalb der kapitalistischen Gesellschaft zu trennen ist. Scharrer/Schütte übersehen dabei jedoch vor allem in Bezug auf ihre abstrakte Leninismuskritik, daß die Forderung nach emanzipativen Elementen innerhalb der Klassenorganisation eine wesentliche Einschränkung erfährt, die nur sehr vermittelt aus dem „Organisationsmodell“ herrührt, sondern vielmehr aus den objektiven Restriktionen des Kampfes, den Repressionen des Klassengegners und seiner Stärke resultiert. Die Möglichkeiten und Methoden des eigenen Kampfes werden so immer vom Gegner mitgeprägt. Eine abstrakte Emanzipationsforderung, die diesen Zusammenhang in ihrer Leninismuskritik nicht einmal erwähnt, gerät damit schlicht zum voluntaristischen Postulat.

5. Der Opportunismusvorwurf gegenüber dem Projekt Klassenanalyse ist moralisch und an keiner Stelle wirklich begründet. Die anscheinend spezifischen Schwierigkeiten des Projekts, sich politisch zu bestimmen, die übrigens auch bei der PROKLA vorhanden sind, gründen in der Geschichte und dem Zerfallsprozeß der Studentenbewegung, den damit zusammenhängenden Desillusionierungen, der relativ stagnierenden Klassenkampfbewegung in der BRD und der spezifischen, vom wirklichen gesellschaftlichen Prozeß abgehobenen Rolle von Intellektuellen, den daraus erwachsenden Sicherheitsbedürfnissen und möglicher Passivität, die nicht denunziatorisch gegen das Projekt gewendet werden können, wenn Scharrer/Schüttes Anspruch nicht unglaubwürdig werden soll, gerade die konkreten Bewußtseinsformen historisch erklären zu wollen, um an ihnen politisch anzuknüpfen.

Die politische Position des Projekts ist so mit der realen historischen Entwicklung der BRD sowie dem Theorieverständnis und der Marxrezeption des Projekts in Zusammenhang zu sehen. Die Schwierigkeit bei der Erklärung dieser Zusammenhänge wird von Scharrer/Schütte mit dem Opportunismusvorwurf zugedeckt. Die Hinwendung zur DKP wird vom Projekt Klassenanalyse damit begründet, daß die DKP angeblich adäquater Ausdruck des Standes der Klassenkämpfe in der BRD ist. Aus seiner von Scharrer/Schütte zu Recht kritisierten mechanistischen Bestimmung des Verhältnisses von Kapitalbewegung, kapitalistischer Vergesellschaftung der Arbeit, Klassenbewußtsein und Partei lehnt das Projekt es daher als sektiererisch ab, wenn sich Intellektuelle von der DKP aufgrund deren reformistischer Ideologiebestandteile und bürokratischer Verfestigungen distarizieren, anstatt im Zusammenhang mit der DKP auf sie kritisch einzuwirken. Daß das Projekt in seinem mechanistischen Objektivismus dabei so gut wie jede Kritik sowohl an der Geschichte als auch der 
jetzigen Praxis der Partei totschweigt und damit die DKP zu immunisieren versucht, erscheint uns politisch gefährlich, besonders dann, wenn das Projekt in seiner denunziatorischen und unsolidarischen Form der Auseinandersetzung mit politischen Gruppen und theoretischen Positionen außerhalb der DKP notwendige politische Diskussionsprozesse zu behindern sucht.

6. Die Redaktionskonferenz stimmt dem Abdruck des Aufsatzes trotz aller kritischen Einwände zu, da er die Möglichkeit bietet, eine überfällige Diskussion innerhalb der PROKLA zu führen. Ein Anfang soll mit einem Aufsatz zur Leninismus-Frage von B. Rabehl in Nr. 11/12 der PROKLA, der sich mit den Thesen von Scharrer/Schütte auseinandersetzt, gemacht werden.

\section{SOZIAL - WISSENSCHAFTLICHE PAPERBACKS}

Rudi Schmiede

Grundprobleme der Marx'schen Akkumulations- und Krisentheorie

1973. ca 200 Seiten, ca. DM 18.-

Christoph Deutschmann

Der linke Keynesianismus

1973, ca. 200 Seiten, ca. DM 18.-

H. C. F. Mansilla

Systembedürfnis und Anpassung 1973, ca. 200 Seiten ca. DM 18.-

\section{QUELLEN UND STUDIEN}

ZUA SOZIALGESCHICHTE

Hans Peter Harstick (Hrsg.)

Karl Marx: Über Formen vorkapitalistischer Produktion

Band 1: Vergleichende Studien zur Geschichte des Grundeigentums. Exzerpte aus M. M. Kovalevskif: Der Gemeindelandsitz (1879). Die Erstausgabe des Originaltextes mit einer Froblem- geschichtlichen Einführung

1974 , ca. 450 Seiten, ca. DM 26.-

\section{SOZIAL.}

WISSENSCHAFTLICHE SKRIPTEN

Dirk Hülsí

Tauschprinzip und antagonistische Gesellschaft

Zum Verhältnis von Konflikt und Iniegration in der "Kritik der politischen Ókonomie" von Karl Marx 1973, ca. 100 Seiten, ca. DM 8.Jürgen Ritsert

Probleme polisisch-ökonomischer Theoriebildung

1973, ca. 160 Seiten, ca. DM 8.-

Mike Rot

Zur Kernstruktur

unserer kapitalistischen Gesellscharl 1972, 102 Seiten, DM 5.-

\section{ATHENA'UM VERLAG}

